\title{
Corneal and Scleral Involvement in Inflammatory Joint Disease: Rheumatologists and Ophthalmologists Exchanging Views
}

Gaëlle Clavel $^{1,2}$, Eric Gabison ${ }^{3}$, Luca Semerrano ${ }^{2,4}$

${ }^{1}$ Service de Médecine Interne, Fondation A. de Rothschild, 25-29 rue Manin, 75019 Paris, France

${ }^{2}$ Inserm UMR 1125, 1, rue de Chablis, 93017 Bobigny, France

${ }^{3}$ Service d'Ophtalmologie, Fondation A. de Rothschild, 25-29 rue Manin, 75019 Paris, France

${ }^{4}$ Service de rhumatologie, Assistance publique-Hôpitaux de Paris (AP-HP), groupe hospitalier Avicenne - Jean-Verdier-René-Muret, 125, rue de Stalingrad, 93017 Bobigny, France

Corresponding author: Gaëlle Clavel

Service de Médecine Interne, Fondation A. de Rothschild, 25-29 rue Manin, 75019 Paris, France

E-mail: gaelle.clavel@gmail.com 


\begin{abstract}
Corneal and scleral disorders related to inflammatory rheumatic diseases vary both in frequency and in severity. Sicca syndrome and its complications are the most common ocular manifestations and, together with episcleritis, can usually be managed by topical treatments. In contrast, the various forms of scleritis and peripheral ulcerative keratitis generally require systemic glucocorticoid therapy and the initiation or intensification of immunosuppressive treatment. Corneal and scleral manifestations are inaugural in a few patients with chronic inflammatory rheumatic disease. No direct information is available on the frequency of severe corneal and scleral involvement, which can only be estimated by extrapolating data from case-series or cohorts, many of which are historical. Similarly, given the absence of randomized controlled trials, treatment decisions must rely on clinical experience acquired in referral centers and on reports of small case-series studies. The rheumatologist and ophthalmologist must work closely together to ensure the prompt and optimal management of these potentially serious conditions.
\end{abstract}

Keywords: Inflammatory joint disease. Rheumatoid arthritis. Scleritis. Peripheral ulcerative keratitis. Treatment. 


\section{The rheumatologist and the ophthalmologist: a duo}

When providing follow-up to patients with chronic inflammatory rheumatic diseases (cIRDs), rheumatologists often detect extraarticular manifestations related to the disease itself or to its treatments. A specific challenge raised by ocular involvement is the difficulty for the rheumatologist to determine which structure is affected and therefore to establish an accurate diagnosis. Ophthalmologists can assess the ocular abnormality in detail and may also provide information of use for reappraising the diagnosis and treatment. However, most ophthalmologists are unfamiliar with the management of the extra-ocular manifestations of cIRDs, notably the use of immunosuppressants. The rheumatologist and ophthalmologist must therefore work in partnership to ensure that the many facets of the disease in each patient receive appropriate attention.

The management of the corneal and scleral manifestations of cIRD is a good model of the fruitful interaction between rheumatologists and ophthalmologists designed to provide patients with an optimal treatment strategy devised during a multidisciplinary approach.

\section{Essential anatomical facts (Figure 1)}

The sclera is the tough white opaque layer that coats most of the outer surface of the eyeball. It receives a blood supply, and its anterior portion is covered by the conjunctiva. The extraocular muscles attach to the sclera. Anteriorly, the sclera transitions to the cornea, which in contrast is transparent and devoid of blood vessels. The cornea serves as a converging lens.

\section{Sicca syndrome}


The most common corneal and scleral manifestation in rheumatoid arthritis (RA) is sicca syndrome related to secondary Sjögren's syndrome [1]. Sicca symptoms arise due to impaired tear-film function caused by a decrease in exocrine lachrymal gland secretion or by alterations in tear composition. These impairments result in excessive evaporation at the surface of the eye and cause tear-film breakup. If not appropriately treated, sicca syndrome can lead to superficial punctate keratitis or filamentary keratitis and, eventually, to corneal ulceration or perforation.

The diagnostic significance and therapeutic implications vary with the type of corneal ulcer. Corneal ulcers related to Sjögren's syndrome are trophic lesions induced by alterations in tear quality and a deficiency in the growth factors normally supplied by tears. Another factor is the local eye surface inflammation caused by severe dryness. The corneal ulcers, known as central or paracentral aseptic keratolysis or corneal melting, share semiological and pathophysiological features with the corneal ulcers seen during graft-versus-host disease (GHVD) [2] and with the iatrogenic corneal ulcers induced by epithelial growth factor receptor (EGFR) antagonists such as erlotinib and gefitinib [3]. They differ from the corneal marginal ulcers that characterize systemic vasculitis (peripheral ulcerative keratitis, PUK), which are directly caused by the intravascular deposition of circulating immune complexes within the terminal marginal corneal vascular arcades (Figure 2).

The treatment aims to protect the cornea by applying topical agents (Box 1). Pilocarpine increases salivary output but has little effect on ocular dryness. Diseasemodifying antirheumatic drugs (DMARDs) have not been proven effective on primary or secondary sicca syndrome [4]. In moderate-to-severe cases, topical ciclosporin in concentrations of $0.05 \%$ to $2 \%$ can be given, in combination with $20 \%$ autologous serum.

\section{Scleritis}


Scleritis is viewed as the most specific ocular manifestation of RA. Scleritis is an umbrella term for a variety of disorders characterized by inflammation involving the sclera and extending in some cases to the episclera, cornea, and uvea. Redness of the sclera with pain develops gradually. Visual acuity may be decreased. Perforation of the sclera is a less common manifestation. Three types of scleritis can be distinguished based on the clinical presentation (Figure 3): anterior scleritis, which may be nodular, diffuse, or necrotizing; noninfectious nonnecrotizing anterior scleritis, which is the most common form; and posterior scleritis, which may raise diagnostic challenges as the eye is painful but not red. The diagnostic and prognostic value of this classification is considerable. Necrotizing scleritis with inflammation is the most severe form and generally occurs in association with a systemic disease, chiefly RA or granulomatosis with polyangiitis (GPA) and, more rarely, spondyloarthritis or atrophic polychondritis (Figure 4). Necrotizing scleritis without inflammation, known as scleromalacia perforans, occurs almost exclusively during advanced RA and is a marker of poor prognosis. Scleritis is associated with severe complications such as PUK, scleromalacia with secondary scleral perforation, and secondary glaucoma. Optical neuropathy and retinal lesions may develop in patients with posterior scleritis.

In a study of 196 patients with RA, 77 (39\%) of patients were found to have ocular manifestations, of which sicca syndrome was the most common $(n=54,28 \%)$ [5]. Scleritis was a feature in only $4(2 \%)$ patients. A strong correlation was noted between the presence of anti-citrullinated peptide antibodies (ACPAs) and ocular manifestations. The prevalence of ocular disease was similar in earlier work [6]. During a prospective study of 107 patients with RA, 9 of the 10 patients with scleritis died during the 8-year follow-up [7]. Thus, scleritis is similar in severity to neurological involvement, skin ulcers, and rheumatoid vasculitis. Although this study was done before the introduction of biotherapies for patients with severe RA, its findings highlight the somber prognostic significance of scleritis. In a 
nonrandomized interventional study of 34 patients who developed ocular manifestations of RA, 17 continued to receive conventional therapy and 17 were also given immunosuppressants [8]. In the conventional treatment group, after 8 years many patients had progression of their ocular disorders and $9(58 \%)$ had died. No deaths occurred in the group given immunosuppressants. This study also antedates the introduction of targeted therapies.

Overall, these data suggest that scleritis and PUK should be viewed as manifestations of subclinical vasculitis that warrant intensification of the systemic treatment for RA, even in the absence of joint findings indicating active disease and/or structural progression [9]. Thus, there is a good level of agreement regarding the management of ocular disease in patients with previous rheumatic or systemic manifestations.

A review of recently published large case-series studies of scleritis showed that the main associated systemic diseases after RA were GPA, spondyloarthropathy, and atrophic polychondritis [10]. Systemic lupus erythematosus (SLE) and inflammatory bowel disease (IBD) were less common. The main toxic agents associated with scleritis were bisphosphonates [10]. Scleritis has been reported as a paradoxical reaction to etanercept therapy but is exceedingly rare, with only 11 reported cases to date [11]. No similar cases have been described with other biotherapies. More specifically, no cases of tocilizumabrelated scleritis have been recorded in the REGATE registry [12].

Importantly, scleritis may develop before the underlying systemic disease is diagnosed. Etiological investigations are therefore in order in patients presenting with apparently isolated ocular manifestations. Close collaboration between the ophthalmologist and the rheumatologist is particularly important in this situation, as the etiological diagnosis can only be provided by the investigations obtained initially or during follow-up [13]. Investigations for systemic diseases must be performed even when there are no signs to provide diagnostic 
orientation. These investigations must be repeated over time if needed. The likelihood of an underlying systemic disease varies with the type of scleritis, from $80 \%$ for necrotizing scleritis to $19 \%$ for posterior scleritis. Scleritis may be the inaugural manifestation of the systemic disease in about one-third of cases [10].

Of 119 patients with scleritis included in a retrospective study, $76.5 \%$ had no evidence of autoimmune disease at the diagnosis of scleritis [13]. Of the 70 patients who underwent rheumatoid factor (RF) assays, 19 had positive results, including 10 who eventually received a diagnosis of RA, compared to a single RF-negative patient. Mean time to diagnosis was 10.6 months (range, 0-72 months). A positive RF assay was associated with an odds ratio (OR) for developing RA of 55.6 (95\% confidence interval [95\%CI], 7.8-398). Of the 70 patients tested for anti-neutrophil cytoplasmic antibody (ANCA), 7 (10\%) were positive, including 3 who were subsequently diagnosed with Wegener's granulomatosis (OR, 22.9; 95\%CI, 3-154). No recent studies have evaluated the value of a positive ACPA test as a predictor of RA in patients with isolated scleritis. Regardless of the situation, scleritis must be viewed as an ocular manifestation of vasculitis [14]. A study of 25 biopsies of necrotizing scleritis and 5 of relapsing scleritis demonstrated fibrinoid vasculitis in $75 \%$ of scleral samples and $50 \%$ of conjunctival samples [15].

\section{Treatment of scleritis}

No randomized trials or evaluations of treatment strategies are available. Data from the available cohorts of patients with scleritis are discordant, in part due to the considerable differences in practices across centers. In addition, many studies are from the pre-biotherapy era.

Whether the patient is known to have an underlying systemic disease has a major influence on the treatment approach to scleritis. In patients receiving follow-up for cIRD, the 
role of the ophthalmologist is to differentiate scleritis from episcleritis, to identify the clinical disease pattern, and to assess severity. The rheumatologist and ophthalmologist can then work together to determine the best initial treatment and to decide whether changes in the DMARD therapy are required (Table 1).

Topical glucocorticoid therapy is the first-line treatment of idiopathic nodular or diffuse scleritis. Subconjunctival triamcinolone injection, although still controversial, may control the inflammatory process, thereby obviating the need for systemic glucocorticoid therapy [16]. If this approach fails, nonsteroidal antiinflammatory drug (NSAID) or oral glucocorticoid therapy should be considered. However, except in the mildest forms, an immunomodulating drug must usually be given, if only to decrease the glucocorticoid requirements. The many synthetic DMARDs used in this indication [1], with variable efficacy, include methotrexate [17], azathioprine [18], mycophenolate mofetil [19], cyclophosphamide [20], and chlorambucil. Most of the available information comes from the vast Systemic Immunosuppressive Therapy for Eye diseases (SITE) cohort study from the US, which evaluated immunosuppressants used in 9250 patients with ocular inflammation [21]. The results failed to provide a ranking of the immunosuppressants according to the nature of the ocular disease. Cyclophosphamide was not superior over other immunosuppressants in a multicenter retrospective study from South Korea but was associated with a higher rate of adverse events [22].

Glucocorticoid-dependent or resistant patients must receive close long-term follow-up. DMARD therapy should be provided to patients with glucocorticoid dependency. Of 86 patients in a retrospective study covering $2001-2007,64 \%$ had isolated scleritis and $93 \%$ received systemic therapy, which included a synthetic DMARD in 37 patients, usually methotrexate or mycophenolate mofetil [23]. Infliximab was used in 5 patients, to good effect. 
Necrotizing scleritis is the most serious form and requires first-line glucocorticoid therapy given intravenously then orally. Topical glucocorticoids are administered as an adjunct. The ophthalmologist must take on a central role when necrotizing scleritis develops in a patient with a known systemic disease. After the emergency phase, the ophthalmologist must discuss DMARD therapy intensification with the rheumatologist or internist.

Targeted treatments are usually considered only after failure of glucocorticoid therapy with or without synthetic DMARD therapy. Their place in the treatment of scleritis, as well as selection of the treatment target, is unclear, as the only available evidence comes from anecdotal case-reports and small case-series studies [24]. The most commonly targeted treatments used are TNF $\alpha$ antagonists (usually infliximab) [25-42] and rituximab [42-51]. Comparisons of studies are hindered by variations in the efficacy criteria used (e.g., disease activity score, maintenance of adjuvant therapy or glucocorticoid therapy, or remission period). Of the two available prospective studies, one is a nonrandomized evaluation of infliximab in 5 patients [25] and the other a randomized phase I/II trial of rituximab in 12 patients with refractory scleritis [43]. An important caveat is that publication bias with failure to publish studies or case-reports showing no therapeutic effect is likely to have occurred.

Tocilizumab has been reported to be effective in a few patients $[52,53]$ but produced somewhat discordant results in the small published case-series studies. Thus, in a retrospective study, of 6 patients with scleritis, all of whom had received several lines of DMARD and/or biological therapy and 4 of whom had RA, 3 responded to tocilizumab. Another study demonstrated good efficacy in 3 patients and partial efficacy in 1 patient, but these data require confirmation as mean follow-up was only 6.5 months (range, 4-11 months) [54]. 
The efficacy of the anti-IL-1 antibody gevokizumab in scleritis has been assessed in a single study, which used a nonrandomized phase I/II design and included 8 patients with anterior scleritis [55]. The primary endpoint was an at least 2-point decrease, or a decrease to 0 , on a 0 to 4 scleral inflammation scale. This endpoint was met within 2 weeks after the first injection in 7 patients. Neither abatacept nor JAK inhibitors have been studied in scleritis. A phase II trial of tofacitinib in idiopathic scleritis and uveitis is scheduled (ClinicalTrials.gov identifier: NCT03580343). The low incidence of scleritis hinders the conduct of randomized trials. Notwithstanding, a multicenter randomized trial comparing infliximab to cyclophosphamide in refractory idiopathic scleritis is scheduled (ClinicalTrials.gov identifier: NCT03088293). Despite the challenges, drug trials in scleritis deserve to be encouraged as crucial sources of information for optimizing patient management.

At present, selection of the treatment relies on the nature of the underlying systemic disease and on the clinical pattern and severity of the scleritis. However, treatment approaches also differ according to the preferences of the rheumatologist/internist and ophthalmologist working in partnership, as well as across treatment centers (Table 1).

\section{Isolated scleritis: key messages}

Mild and severe forms of idiopathic scleritis should be distinguished. Necrosis and/or corneal involvement characterize the severe forms. Progression toward a systemic disease is the rule in patients with severe scleritis. Consequently, close long-term follow-up including repeated investigations for an underlying disease must be provided. In patients with mild but relapsing scleritis, an ocular surface disorder such as posterior blepharitis (which may be related to rosacea) should be considered, as a prolonged remission can only be induced by specific ophthalmological therapy. 
Severe idiopathic unilateral scleritis requires routine investigation for herpes-simplex virus or varicella-zoster virus infection and may warrant a routine trial of antiviral therapy [56]. The most typical presentation of ocular herpes is the development in a patient older than 50 years of UNILATERAL nodular or necrotizing anterior scleritis, with or without lesions of the adjacent cornea. Investigations for systemic disease remain negative, despite the severity and glucocorticoid-dependency of the ocular manifestations. As a general rule, severe idiopathic inflammation affecting a single eye should suggest an infection or a tumor. For instance, conjunctival carcinoma or lymphoma may present as masquerade syndromes that mimic inflammatory disease. The diagnosis requires a slit-lamp examination and, in many cases, a biopsy.

\section{Episcleritis}

Episcleritis differs clinically from scleritis by the absence of pain. Most patients experience only some measure of discomfort (Table 2). The symptoms are more marked in nodular episcleritis than in simple episcleritis. Classically, only the nodular form requires etiological investigations similar to those performed in scleritis. In simple episcleritis, an etiological workup is necessary only in the event of multiple relapses. An ocular surface disorder (e.g., ocular dryness or an allergy) should be sought since, as with scleritis, specific treatment is required to ensure its resolution. Sjögren's syndrome should be ruled out in patients with sicca syndrome.

\section{Peripheral ulcerative keratitis (PUK)}

The presenting symptoms are ocular pain, redness, and photophobia. The peripheral cornea is thinned and opaque. Corneal perforation and visual loss are the most serious complications [57]. Whereas the center of the cornea is avascular, the peripheral cornea or 
limbus receives blood via the corneal vascular arcade, whose vessels are terminal (Figure 5). Inflammatory-cell infiltration of the vessel walls and intraluminal deposition of circulating immune complexes jeopardize the blood supply to the limbus. The first abnormality often consists in a peripheral inflammatory infiltrate, with secondary ulceration. Without appropriate treatment, the lesions progress circumferentially then centripetally and may cause perforation.

As with scleritis, PUK is often associated with an underlying systemic disease. RA is the most common, followed by SLE and GPA [58,59]. PUK with no evidence of scleritis and no systemic disease is termed Mooren's ulcer. The diagnosis of Mooren's ulcer is one of exclusion and illustrates the collaboration between the ophthalmologist, who diagnoses the corneal condition, and the rheumatologist, who establishes that it is idiopathic. The form of PUK seen in elderly individuals, predominantly Caucasians, responds to topical therapy. Another clinical pattern affects younger patients, predominantly Africans, often bilaterally, and usually requires systemic therapy with glucocorticoids or even immunosuppressants. These severe forms that require aggressive treatment raise the issues inherent in the use of immunosuppressants to treat idiopathic conditions. The ophthalmologist determines the indication for immunosuppressive therapy, monitors the patient, and seeks input from the rheumatologist or internist to initiate the treatment (Figure 6). We have reported that rituximab was effective in patients with Mooren's ulcers refractory to glucocorticoids and conventional immunosuppressants such as cyclophosphamide [60]. If present also, scleritis dictates the treatment strategy, and the development of PUK is of adverse prognostic significance.

Topical glucocorticoid therapy is the first-line treatment of PUK. Systemic treatment identical to that used for necrotizing scleritis is required in patients with glucocorticoid resistance (thinning or progression of the inflammatory infiltrate) or glucocorticoid 
dependency, as well as in the event of corneal surgery (grafting of amniotic membrane or peripheral cornea) [57]. As with scleritis, the presence of an underlying autoimmune disease often requires systemic therapy including high-dose glucocorticoids and an immunosuppressant or biological agent. Selection of the drugs is guided by the nature of the autoimmune disease, the treatments used in the past, and the comorbidity profile. Closely spaced ophthalmological examinations are indispensable to guide treatment adjustments.

\section{Concluding remarks}

The spectrum of corneoscleral manifestations of cIRD encompasses sicca syndrome and its complications, which are the most common; episcleritis, which is common but not very severe; and scleritis and PUK, which are medical emergencies. The development of scleral inflammation requires the coordinated intervention of an ophthalmologist and a rheumatologist or internist. The dialogue between these two professionals has three main objectives. First, the optimal treatment of the acute episode must be determined, based chiefly on the severity of the ocular condition, on whether relapses occur, and on the treatments already under way in the event of a known underlying cIRD. The second objective is selection of the investigations needed to establish the diagnosis in patients with isolated ocular manifestations, in the absence of a previous diagnosis of systemic inflammatory disease. This task falls chiefly to the rheumatologist, although the ophthalmologist must first rule out an infectious cause such as herpes virus scleritis. The third objective is to determine the optimal long-term management strategy in patients with known cIRD. The development of scleritis constitutes a turning point in the disease, toward greater severity, and therefore requires intensification of the treatment regimen.

Direct evidence on the prevalence and incidence of scleritis is lacking. Historical caseseries studies suggest that RA may contribute most cases of scleritis associated with cIRD. 
Whether recent therapeutic changes including the earlier administration of more aggressive treatment regimens and the introduction of new drugs for RA will affect the frequency of scleritis is unknown. The creation of nationwide scleritis registries would shed light on changes in the epidemiology of this rare disease. Today, the management of scleritis still varies across centers, reflecting the lack of data from controlled trials. The findings from the scheduled randomized controlled trial of infliximab versus cyclophosphamide is the first step toward an evidence-based approach in this area.

In the current era of hyperspecialization in medicine, the needs of patients with inflammatory disorders of the cornea and sclera underscores the importance of cooperation among the various healthcare professionals involved to ensure that the therapeutic strategy is tailored to each individual.

\section{Disclosure of interest}

The authors have no conflicts of interest to declare. 


\section{References}

[1] Bielefeld P, Saadoun D, Héron E, Abad S, Devilliers H, Deschasse C, et al. [Scleritis and systemic diseases: What should know the internist?]. Rev Med Interne 2018;39:711-20. [2] Qiu Y, Hong J, Peng R. Manifestation of clinical categories of ocular graft-versushost disease. J Ophthalmol 2018;2018:6430953.

[3] Ho WL, Wong H, Yau T. The ophthalmological complications of targeted agents in cancer therapy: what do we need to know as ophthalmologists? Acta Ophthalmol 2013; 91:604-9.

[4] Letaief H, Lukas C, Barnetche T, Gaujoux-Viala C, Combe B, Morel J. Efficacy and safety of biological DMARDs modulating B cells in primary Sjögren's syndrome: Systematic review and meta-analysis. Joint Bone Spine 2018;85:15-22.

[5] Vignesh AP, Srinivasan R. Ocular manifestations of rheumatoid arthritis and their correlation with anti-cyclic citrullinated peptide antibodies. Clin Ophthalmol 2015;9:393-7. [6] Smith JR, Mackensen F, Rosenbaum JT. Therapy insight: scleritis and its relationship to systemic autoimmune disease. Nat Clin Pract Rheumatol 2007;3:219-26.

[7] Erhardt CC, Mumford PA, Venables PJ, Maini RN. Factors predicting a poor life prognosis in rheumatoid arthritis: an eight year prospective study. Ann Rheum Dis 1989;48:7-13.

[8] Foster CS, Forstot SL, Wilson LA. Mortality rate in rheumatoid arthritis patients developing necrotizing scleritis or peripheral ulcerative keratitis. Effects of systemic immunosuppression. Ophthalmology 1984;91:1253-63.

[9] Foster CS. Ocular manifestations of the potentially lethal rheumatologic and vasculitic disorders. J Fr Ophtalmol 2013; 36:526-32.

[10] Héron E, Bourcier T. [Scleritis and episcleritis]. J Fr Ophtalmol 2017; 40:681-95.

[11] Gaujoux-Viala C, Giampietro C, Gaujoux T, Ea H-K, Prati C, Orcel P, et al. Scleritis: 
a paradoxical effect of etanercept? Etanercept-associated inflammatory eye disease. J Rheumatol 2012; 39:233-9.

[12] Terreaux W, Masson C, Eschard J-P, Bardin T, Constantin A, Le Dantec L, et al. Incidence of paradoxical reactions in patients treated with tocilizumab for rheumatoid arthritis: Data from the French registry REGATE. Joint Bone Spine 2018;85:53-7.

[13] Lin P, Bhullar SS, Tessler HH, Goldstein DA. Immunologic markers as potential predictors of systemic autoimmune disease in patients with idiopathic scleritis. Am J Ophthalmol 2008;145:463-71.

[14] Wakefield D, Di Girolamo N, Thurau S, Wildner G, McCluskey P. Scleritis: Immunopathogenesis and molecular basis for therapy. Prog Retin Eye Res 2013;35:44-62. [15] Fong LP, Sainz de la Maza M, Rice BA, Kupferman AE, Foster CS. Immunopathology of scleritis. Ophthalmology 1991;98:472-9.

[16] Beardsley RM, Suhler EB, Rosenbaum JT, Lin P. Pharmacotherapy of scleritis: current paradigms and future directions. Expert Opin Pharmacother 2013;14:411-24. [17] Gangaputra S, Newcomb CW, Liesegang TL, Kaçmaz RO, Jabs DA, Levy-Clarke GA, et al. Methotrexate for ocular inflammatory diseases. Ophthalmology 2009;116:21882198.e1.

[18] Pasadhika S, Kempen JH, Newcomb CW, Liesegang TL, Pujari SS, Rosenbaum JT, et al. Azathioprine for ocular inflammatory diseases. Am J Ophthalmol 2009;148:500509.e2.

[19] Daniel E, Thorne JE, Newcomb CW, Pujari SS, Kaçmaz RO, Levy-Clarke GA, et al. Mycophenolate mofetil for ocular inflammation. Am J Ophthalmol 2010;149:423-432.e1-2. [20] Pujari SS, Kempen JH, Newcomb CW, Gangaputra S, Daniel E, Suhler EB, et al. Cyclophosphamide for ocular inflammatory diseases. Ophthalmology 2010;117:356-65. [21] Kempen JH, Daniel E, Gangaputra S, Dreger K, Jabs DA, Kaçmaz RO, et al. 
Methods for identifying long-term adverse effects of treatment in patients with eye diseases: the Systemic Immunosuppressive Therapy for Eye Diseases (SITE) Cohort Study. Ophthalmic Epidemiol 2008;15:47-55.

[22] Jeon HS, Hyon JY, Kim MK, Chung T-Y, Yoon KC, Kim JY, et al. Efficacy and safety of immunosuppressive agents in the treatment of necrotising scleritis: a retrospective, multicentre study in Korea. British Journal of Ophthalmology 2016;100:1066-70.

[23] Raiji VR, Palestine AG, Parver DL. Scleritis and systemic disease association in a community-based referral practice. Am J Ophthalmol 2009;148:946-50.

[24] de Fidelix TS de A, Vieira LA, de Freitas D, Trevisani VFM. Biologic therapy for refractory scleritis: a new treatment perspective. Int Ophthalmol 2015;35:903-12.

[25] Sen HN, Sangave A, Hammel K, Levy-Clarke G, Nussenblatt RB. Infliximab for the treatment of active scleritis. Can J Ophthalmol 2009;44:e9-12.

[26] Smith JR, Levinson RD, Holland GN, Jabs DA, Robinson MR, Whitcup SM, et al. Differential efficacy of tumor necrosis factor inhibition in the management of inflammatory eye disease and associated rheumatic disease. Arthritis Rheum 2001;45:252-7.

[27] Bawazeer AM, Raffa LH. Adalimumab in the treatment of recurrent idiopathic bilateral nodular scleritis. Oman J Ophthalmol 2011;4:139-41.

[28] Lawuyi LE, Gurbaxani A. Refractory necrotizing scleritis successfully treated with adalimumab. J Ophthalmic Inflamm Infect 2016;6:37.

[29] Ragam A, Kolomeyer AM, Fang C, Xu Y, Chu DS. Treatment of chronic, noninfectious, nonnecrotizing scleritis with tumor necrosis factor alpha inhibitors. Ocul Immunol Inflamm 2014;22:469-77.

[30] Murphy CC, Ayliffe WH, Booth A, Makanjuola D, Andrews PA, Jayne D. Tumor necrosis factor alpha blockade with infliximab for refractory uveitis and scleritis. Ophthalmology 2004;111:352-6. 
[31] Cazabon S, Over K, Butcher J. The successful use of infliximab in resistant relapsing polychondritis and associated scleritis. Eye (Lond) 2005;19:222-4.

[32] El-Shabrawi Y, Hermann J. Anti-TNF alpha therapy in chronic necrotizing scleritis resistant to standard immunomodulatory therapy in a patient with Wegener's granulomatosis. Eye (Lond) 2005;19:1017-8.

[33] Ashok D, Ayliffe WH, Kiely PD. Necrotizing scleritis associated with rheumatoid arthritis: long-term remission with high-dose infliximab therapy. Rheumatology (Oxford) 2005;44:950-1.

[34] Atchia II, Kidd CE, Bell RW. Rheumatoid arthritis-associated necrotizing scleritis and peripheral ulcerative keratitis treated successfully with infliximab. J Clin Rheumatol 2006;12:291-3.

[35] Weiss K, Rieger R, Keitzer R, Pleyer U. Successful infliximab treatment of posterior scleritis in a 13-year-old child refractory to other immunosuppressive therapy. Graefes Arch Clin Exp Ophthalmol 2007;245:1735-7.

[36] Sobrin L, Kim EC, Christen W, Papadaki T, Letko E, Foster CS. Infliximab therapy for the treatment of refractory ocular inflammatory disease. Arch Ophthalmol 2007;125:895-900.

[37] Ahn SJ, Oh JY, Kim MK, Wee WR. Treating refractory scleritis with infliximab. Jpn J Ophthalmol 2009;53:286-7.

[38] Kontkanen M, Paimela L, Kaarniranta K. Regression of necrotizing scleritis in Wegener's granulomatosis after infliximab treatment. Acta Ophthalmol 2010;88:e96-97. [39] Herrera-Esparza R, Avalos-Díaz E. Infliximab treatment in a case of rheumatoid scleromalacia perforans. Reumatismo 2009;61:212-5.

[40] Jabbarvand M, Fard MA. Infliximab in a patient with refractory necrotizing scleritis associated with relapsing polychondritis. Ocul Immunol Inflamm 2010;18:216-7. 
[41] Doctor P, Sultan A, Syed S, Christen W, Bhat P, Foster CS. Infliximab for the treatment of refractory scleritis. Br J Ophthalmol 2010;94:579-83.

[42] Morarji J, Joshi L, Tomkins-Netzer O, Lightman S, Taylor SR. Combined infliximab and rituximab in necrotising scleritis. Case Rep Ophthalmol 2012;3:286-90.

[43] Suhler EB, Lim LL, Beardsley RM, Giles TR, Pasadhika S, Lee ST, et al. Rituximab therapy for refractory scleritis: results of a phase I/II dose-ranging, randomized, clinical trial. Ophthalmology 2014;121:1885-91.

[44] Bogdanic-Werner K, Fernandez-Sanz G, Alejandre Alba N, Ferrer Soldevila P, Romero-Bueno FI, Sanchez-Pernaute O. Rituximab therapy for refractory idiopathic scleritis. Ocul Immunol Inflamm 2013;21:329-32.

[45] Chauhan S, Kamal A, Thompson RN, Estrach C, Moots RJ. Rituximab for treatment of scleritis associated with rheumatoid arthritis. Br J Ophthalmol 2009;93:984-5.

[46] Taylor SRJ, Salama AD, Joshi L, Pusey CD, Lightman SL. Rituximab is effective in the treatment of refractory ophthalmic Wegener's granulomatosis. Arthritis Rheum 2009;60:1540-7.

[47] Kurz PA, Suhler EB, Choi D, Rosenbaum JT. Rituximab for treatment of ocular inflammatory disease: a series of four cases. Br J Ophthalmol 2009;93:546-8.

[48] Joshi L, Tanna A, McAdoo SP, Medjeral-Thomas N, Taylor SRJ, Sandhu G, et al. Long-term outcomes of rituximab therapy in ocular granulomatosis with polyangiitis: Impact on localized and nonlocalized disease. Ophthalmology 2015;122:1262-8.

[49] Recillas-Gispert C, Serna-Ojeda JC, Flores-Suárez LF. Rituximab in the treatment of refractory scleritis in patients with granulomatosis with polyangiitis (Wegener's). Graefes Arch Clin Exp Ophthalmol 2015;253:2279-84.

[50] You C, Ma L, Lasave AF, Foster CS. Rituximab induction and maintenance treatment in patients with scleritis and granulomatosis with polyangiitis (Wegener's). Ocul 
Immunol Inflamm 2017:1-8. doi:10.1080/09273948.2017.1327602.

[51] Cao JH, Oray M, Cocho L, Foster CS. Rituximab in the treatment of refractory noninfectious scleritis. Am J Ophthalmol 2016;164:22-8.

[52] Tode J, Zeuner R, Saeger M, Roider J, Nölle B. Tocilizumab and steroid boli for treatment-resistant anterior necrotizing scleritis. Acta Ophthalmologica 2015;93:e683-4.

[53] Shimizu H, Nishioka H. Successful treatment with tocilizumab for refractory scleritis associated with relapsing polychondritis. Scand J Rheumatol 2017;46:418-9.

[54] Sené T, Clavel-Refregiers G, Titah C, Cochereau I, Ziza JM. Efficacité du tocilizumab dans les sclérites non nécrosantes réfractaires. À propos de 4 cas. La Revue de Médecine Interne 2018;39:A204-5.

[55] Knickelbein JE, Tucker WR, Bhatt N, Armbrust K, Valent D, Obiyor D, et al. Gevokizumab in the treatment of autoimmune non-necrotizing anterior scleritis: Results of a phase I/II clinical trial. Am J Ophthalmol 2016;172:104-10.

[56] Bhat PV, Jakobiec FA, Kurbanyan K, Zhao T, Foster CS. Chronic herpes simplex scleritis: characterization of 9 cases of an underrecognized clinical entity. Am J Ophthalmol 2009;148:779-789.e2.

[57] Cao Y, Zhang W, Wu J, Zhang H, Zhou H. Peripheral ulcerative keratitis associated with autoimmune disease: Pathogenesis and treatment. J Ophthalmol 2017;2017:7298026. [58] Murray PI, Rauz S. The eye and inflammatory rheumatic diseases: The eye and rheumatoid arthritis, ankylosing spondylitis, psoriatic arthritis. Best Pract Res Clin Rheumatol 2016;30:802-25.

[59] Artifoni M, Rothschild PR, Brézin A, Guillevin L, Puéchal X. Ocular inflammatory diseases associated with rheumatoid arthritis. Nat Rev Rheumatol 2014;10:108-16.

[60] Guindolet D, Reynaud C, Clavel G, Belangé G, Benmahmed M, Doan S, et al. Management of severe and refractory Mooren's ulcers with rituximab. Br J Ophthalmol 
2017;101:418-22. 


\section{FIGURE LEGENDS}

Figure 1: Main anatomical structures

Figure 2: Inflammatory keratitis

(A) Peripheral ulcerative keratitis (PUK) is usually related to systemic vasculitis. The arrows point to a peripheral corneal ulcer; (B) Aseptic paracentral keratolysis (*) complicating severe dryness in a patient with graft-versus-host disease

Figure 3: Clinical classification of anterior scleritis (A) Diffuse scleritis; (B) Nodular scleritis; magnified view on the right; (C) Necrotizing scleritis with inflammation (arrows); (D) Necrotizing scleritis without inflammation (scleromalacia perforans: arrows)

Figure 4: Diffuse anterior scleritis in a patient with atrophic polychondritis Note the nasal and auricular changes due to chondritis.

(A) Diffuse anterior scleritis; (B) Peripheral corneal ulcer (peripheral immune keratitis) ;

(C) Blue light photograph after fluorescein instillation: peripheral corneal infiltrate

Figure 5: Anterior necrotizing scleritis with inflammation

Green light examination of the ischemic zones and favorable angiogenic response (arrows) after the initiation of immunosuppressant therapy

Figure 6: Pathophysiological model of peripheral ulcerative keratitis (PUK) 
Box 1: Treatment of sicca syndrome

\section{Early ocular dryness related to dysimmunity}

Discontinuation of potentially harmful topical treatments (all eye drops containing preservatives, antiseptics, antibiotics, or nonsteroidal antiinflammatory drugs)

Moisturizing eye drops

- Methylcellulose (e.g., Celluvisc $\left.{ }^{\circledR}\right)$, hyaluronic acid (e.g., Vismed $\left.{ }^{\circledR}\right)$ (1 every 4 hours up to 1 per hour)

- Meatus plugs (preferably four) OPH

Moderate-to-severe ocular dryness (superficial punctate keratitis)

- Add-on topical cyclosporine (Ikervis ${ }^{\circledR}$ or preparation by the pharmacist; chiefly a maintenance treatment, with delayed effects) $\mathrm{OPH}$

- Brief course of topical glucocorticoids (fluorometholone ,FLUCON ${ }^{\circledR}$; or dexamethasone, DEXAFREE $^{\circledR}$; useful at cyclosporine therapy initiation to improve tolerance and accelerate the response) $\mathrm{OPH}$

- $20 \%$ autologous serum (prepared at hospital pharmacies) if persistent superficial punctate keratitis or aseptic central keratolysis. OPH

- Scleral contact lenses (improve vision and quality of life in patients with severe dryness) $\mathrm{OPH}$

OPH: to be performed by an ophthalmologist 
Table 1: Management of scleritis

\section{Treatment of scleritis}

\begin{tabular}{|c|c|c|c|}
\hline \multicolumn{4}{|c|}{ Treatment of scleritis } \\
\hline \multicolumn{2}{|c|}{ Known systemic disease } & \multicolumn{2}{|c|}{$\begin{array}{l}\text { No known etiology: investigations for a cause } \\
\text { (repeat as needed) }\end{array}$} \\
\hline \multicolumn{4}{|c|}{ Evaluate the severity of the ocular condition: clinical pattern, complications } \\
\hline Mild & Severe & Mild & Severe \\
\hline Topical treatment & $\begin{array}{l}\text { Systemic GCs } \\
\text { Intensify DMARD } \\
\text { therapy: IS, biotherapy }\end{array}$ & Topical treatment & First-line systemic GCs \\
\hline $\begin{array}{l}\text { If failure: change the } \\
\text { DMARD regimen and add } \\
\text { systemic GCs }\end{array}$ & & $\begin{array}{l}\text { If failure: systemic } \\
\text { treatment }\end{array}$ & \begin{tabular}{|l|} 
If failure or GC \\
dependency: \\
Subconjunctival \\
triamcinolone injection / \\
IS or biotherapy \\
\end{tabular} \\
\hline \multicolumn{2}{|c|}{$\begin{array}{l}\text { Selection of DMARDs according to the underlying } \\
\text { disease: RA, GPA, ... }\end{array}$} & \multicolumn{2}{|c|}{$\begin{array}{l}\text { IS: Azathioprine, methotrexate, ... } \\
\text { Biotherapy: AT anti-TNF, rituximab, anti-IL-6,.. }\end{array}$} \\
\hline \multicolumn{4}{|c|}{ Ophthalmological follow-up: dictates treatment adjustments } \\
\hline \multicolumn{4}{|c|}{ Overall follow-up: treatment safety + extra-ophthalmological activity } \\
\hline
\end{tabular}

GC, glucocorticoids; DMARD, disease-modifying antirheumatic drug; IS, immunosuppressant; RA, rheumatoid arthritis; GPA, granulomatosis with polyangiitis 
Table 2: Distinguishing between scleritis and episcleritis

\begin{tabular}{|l|l|}
\hline Episcleritis & Scleritis \\
\hline Daylight examination: red & Dark purplish red \\
\hline Sudden onset & Gradual onset \\
\hline Spontaneous resolution possible & Worsens gradually without treatment \\
\hline Common, idiopathic $>60 \%$ & Rare, systemic disease $>50 \%$ \\
\hline No pain, ocular discomfort & $\begin{array}{l}\text { Gradually worsening pain with insomnia, } \\
\text { aggravated by eyeball movements and palpation }\end{array}$ \\
\hline$<30$ years of age & $>40$ years of age \\
\hline $\begin{array}{l}\text { Redness resolves within } 30 \mathrm{~s} \text { after phenylephrine } \\
\text { instillation }\end{array}$ & $\begin{array}{l}\text { Redness does not resolve after phenylephrine } \\
\text { instillation }\end{array}$ \\
\hline
\end{tabular}


Figure 1

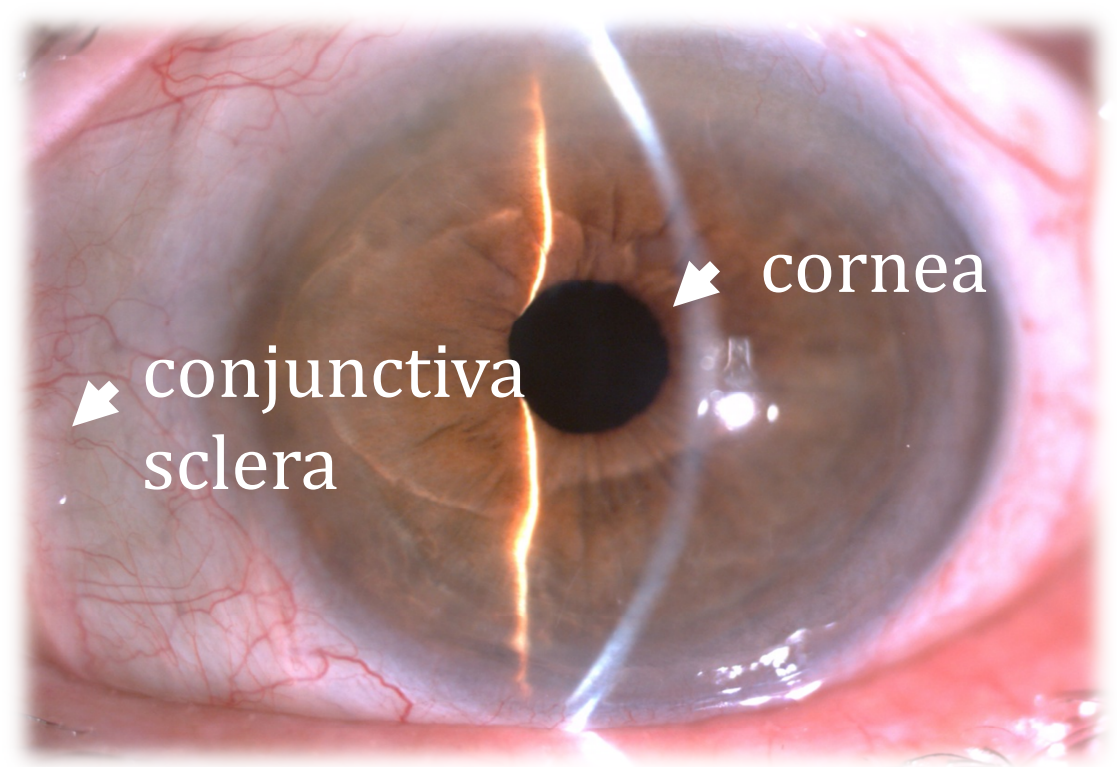


Figure 2
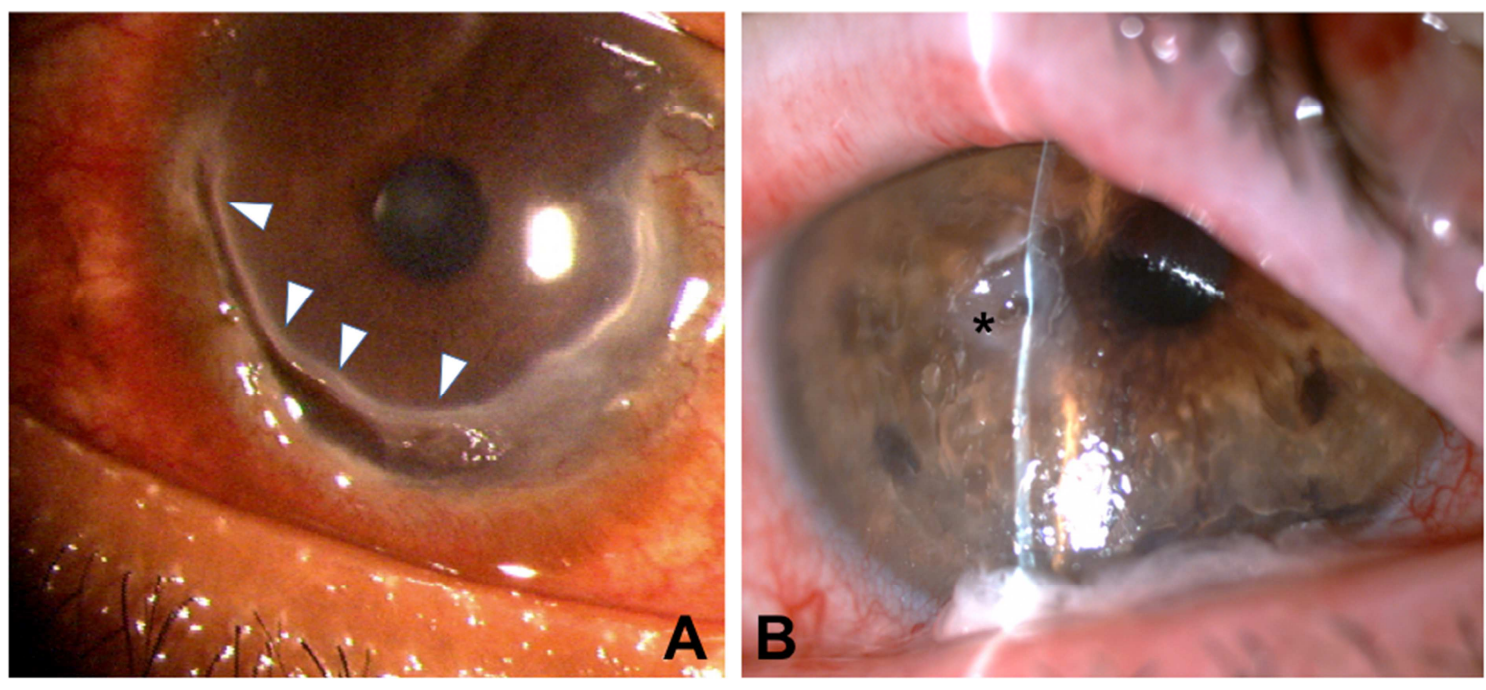
Figure 3
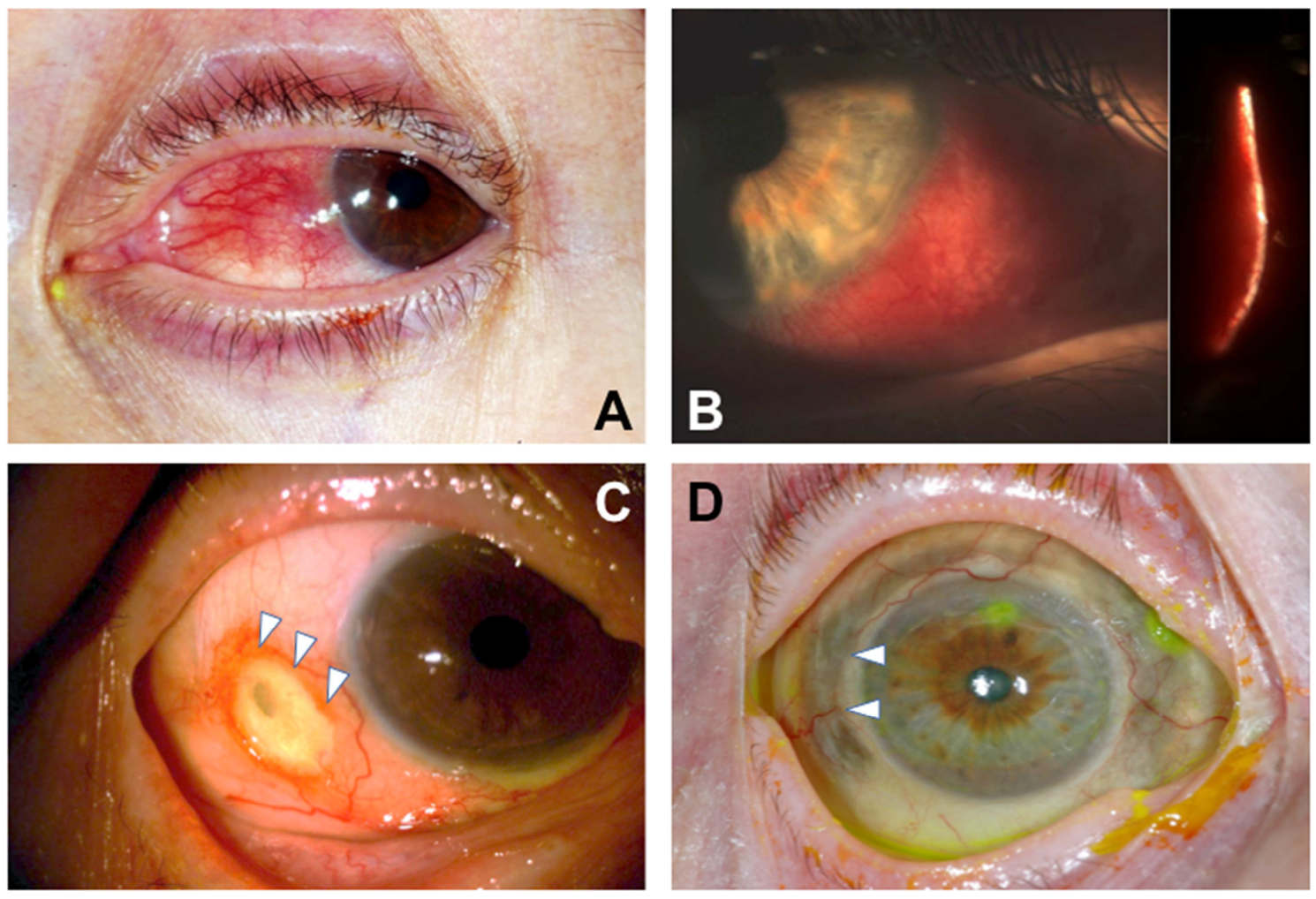
Figure 4
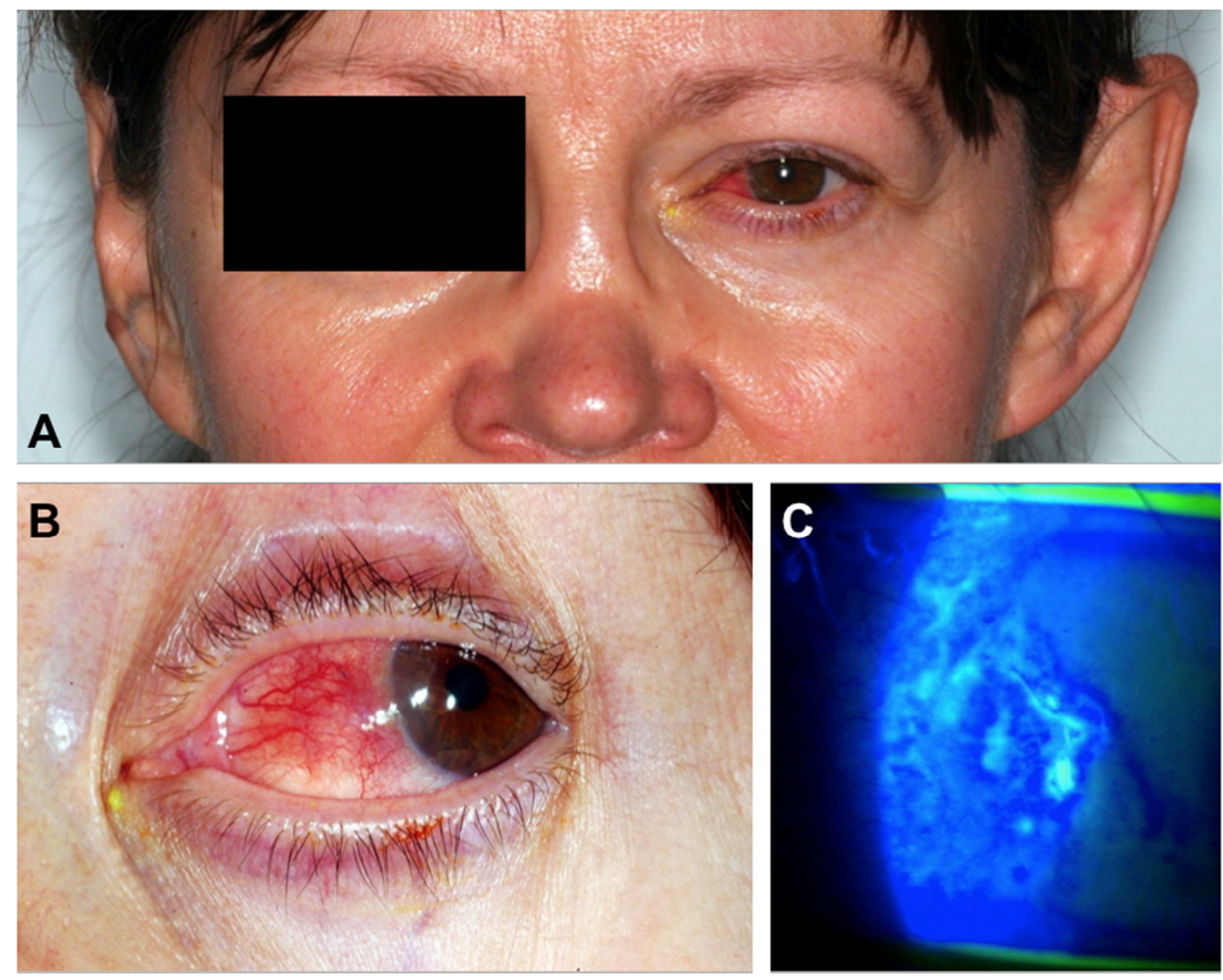
Figure 5
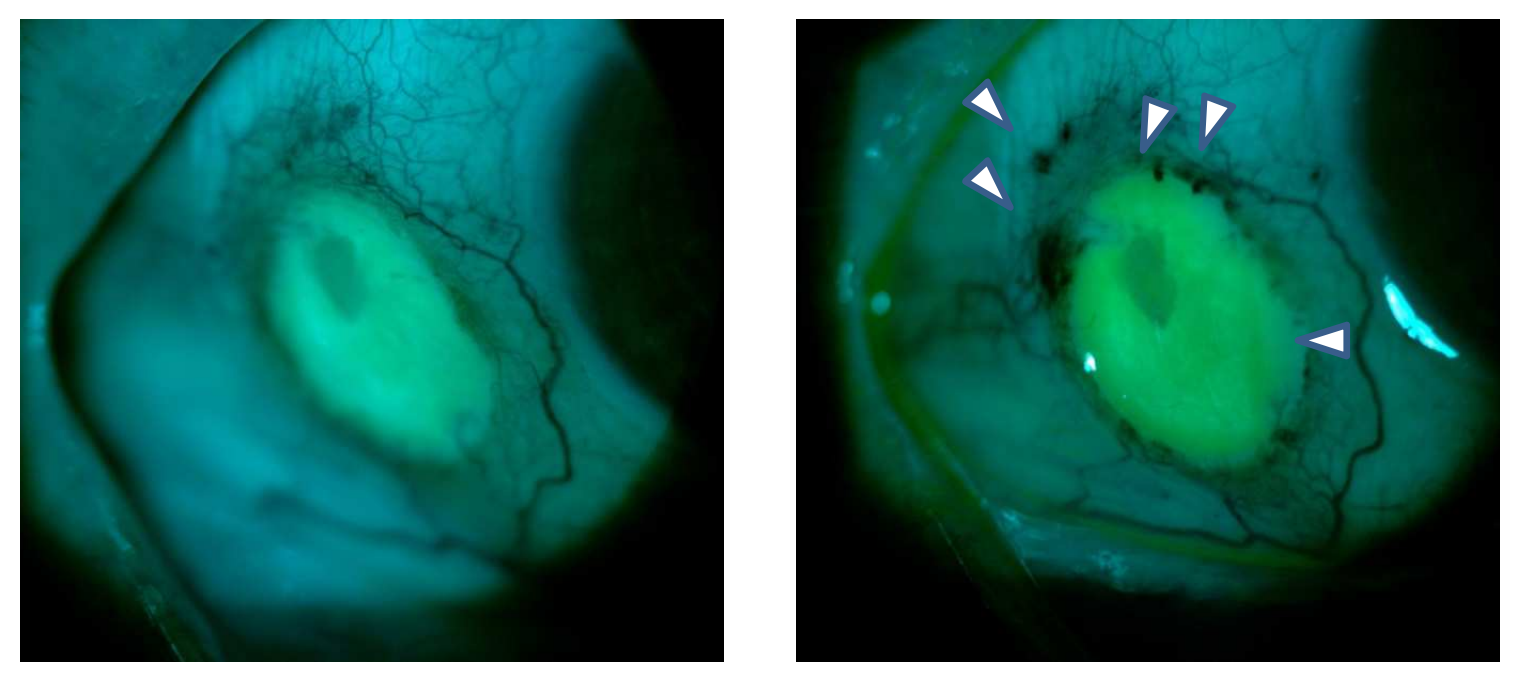


\section{Peripheral corneal inflammation}

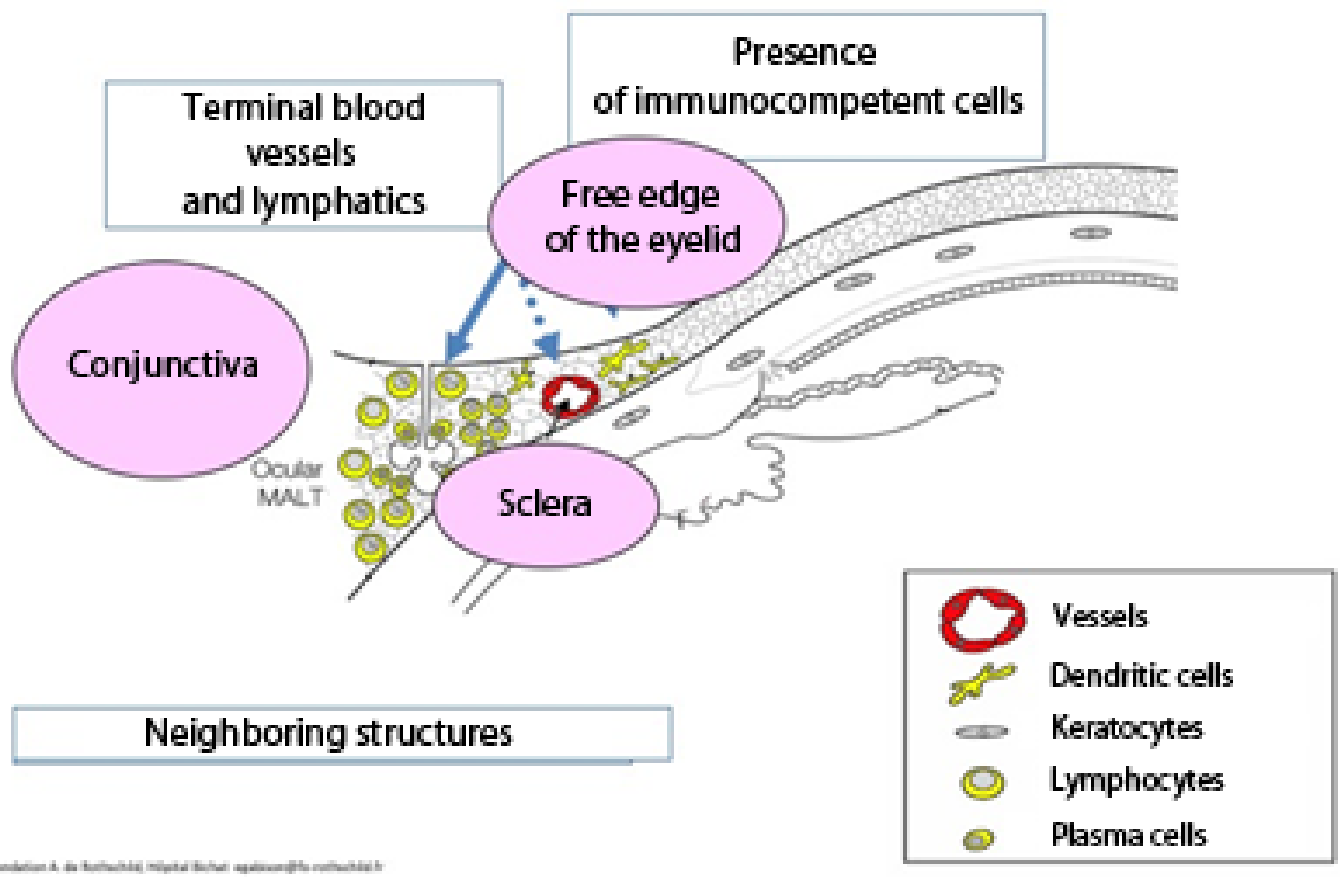

